\title{
HERRAMIENTAS TIC Y SOFTWARE ESPECÍFICO. UN BUEN COMPLEMENTO EN EL AULA UNIVERSITARIA.
}

\author{
Artal-Sevil J.S. a , Peña Pellicer B. ${ }^{\text {b }}$, Luesma Bartolomé M.J. ${ }^{\text {c }}$ y Gargallo Castel A.F. ${ }^{\text {d }}$ \\ ${ }^{a}$ Dpto. Ingeniería Eléctrica. Escuela de Ingeniería y Arquitectura. Universidad de Zaragoza. \\ Campus Río Ebro. Zaragoza (Spain). email: jsartal@unizar.es. \\ ${ }^{\mathrm{b}}$ Dpto. Ingeniería Mecánica. Escuela de Ingeniería y Arquitectura. Universidad de Zaragoza. \\ Campus Río Ebro. Zaragoza (Spain). email: bpp@unizar.es. \\ ${ }^{\text {c }}$ Dpto. Anatomía e Histología Humanas. Facultad de Ciencias. Universidad de Zaragoza. \\ Campus San Francisco. Zaragoza (Spain). email: mjluesma@,unizar.es. \\ ${ }^{\mathrm{d}}$ Departamento de Dirección y Organización de Empresas. Facultad de Ciencias Sociales y Humanas \\ de Teruel. Universidad de Zaragoza. Teruel (Spain). email: gargallo@unizar.es.
}

\begin{abstract}
This document presents an educational methodology Simulation-based learning (SbL) centered on the student through the application of an activecollaborative strategy. The incorporation of technology in the classroom has allowed to change the way in which students interact with information and content. The strategy is based on the use of specific software supplemented with a series of free ICT tools. The results show how the methodology has two main advantages. On the one hand, it facilitates obtaining technical and personal skills. While on the other hand the students solve real problems. The objective has been to show an interdisciplinary experience within the Flipped Classroom pedagogical approach.
\end{abstract}

Keywords: Flipped Classroom, Blended-Learning, ICT Tools, Educational resources and Interactive tools, Specific software, smartphone, tablet, free software, learning by competences.

\section{Resumen}

En este documento se presenta una metodología educativa Simulation-based Learning (SbL) centrada en el estudiante mediante la aplicación de una estrategia activa-colaborativa. La introducción de la tecnología en las aulas ha permitido cambiar la forma en la que los estudiantes interactúan con la información y contenidos. La estrategia está basada en el uso de software especifico complementado con una serie de herramientas TIC gratuitas. Los resultados muestran cómo la metodología posee dos ventajas principales. Por un lado facilita la obtención de competencias técnicas y personales. Mientras que por otro lado los estudiantes resuelven problemas reales. El objetivo ha sido mostrar una experiencia interdisciplinar dentro del enfoque pedagógico Flipped Classroom.

Palabras clave: Flipped Classroom, Blended-Learning, Herramientas TIC, Recursos educativos y Herramientas interactivas, software especifico, smartphone, tablet, software gratuito, Aprendizaje por Competencias. 


\section{Introducción}

Hoy en día, nadie discute que la introducción de las TIC en la enseñanza universitaria ha proporcionado muchas ventajas y beneficios sobre la educación. La incorporación de la tecnología en las aulas ha permitido cambiar la forma en la que los estudiantes interactúan con la información y contenidos. En todas sus variantes, las tecnologías educativas están siendo un instrumento muy valioso para la enseñanza (Artal-Sevil et al., 2015). También es necesario especificar el gran número de aplicaciones que existen en el mercado destinadas a estos fines. Por lo que resulta conveniente desarrollar un análisis detallado para seleccionar aquellas que resultan más útiles, eficaces y que mejor se adaptan al entorno educativo.

Ahora bien, ¿es posible aplicar nuevos enfoques metodológicos apoyados en recursos tecnológicos que hoy en día existen?, ¿nos ayudan las herramientas TIC a hacer nuestras clases más interactivas, colaborativas y amenas? Apostar por la metodología Flipped Classroom, combinándola con otras técnicas basadas en el Blended-Learning, Puzzle-Based Learning, Game-Based Learning, Problem-Based Learning, Case-Method, Role-Playing,... y usar determinadas herramientas y aplicaciones TIC puede ayudar a contestar a esta serie de cuestiones (Artal-Sevil et al., 2017b), (Toppo, 2011).

La paulatina incorporación de herramientas TIC en el aula ha propiciado que los estudiantes puedan acceder fácilmente a todos los recursos educativos necesarios, ya sean archivos, vídeos, presentaciones o documentos de apoyo multimedia desde sus dispositivos móviles. Obteniéndose de este modo un adecuado aprovechamiento de los contenidos (Jonsson, 2015). Con ellas se ha pretendido lograr un aprendizaje más activo e interactivo. El uso de estas herramientas ha supuesto un impacto evidente en los estudiantes y en su proceso educativo en general. Este hecho las ha convertido en recursos con un gran potencial para la educación universitaria (Touron et al., 2014).

Ahora bien, la irrupción en estos últimos años de gran cantidad de aplicaciones TIC ha hecho a los docentes replantearse el uso de otras aplicaciones o software específicos que se venían utilizando en el aula o laboratorio hasta ese momento. Hay que recordar que en la mayoría de las ocasiones estas herramientas TIC son utilizadas por el profesor sólo como medio para crear un ambiente más dinámico, participativo e interactivo en el aula. Es innegable que son un buen aliado del profesor en la consecución de los objetivos de aprendizaje (Artal-Sevil et al., 2017). Aunque no hay que pasar por alto, ni tampoco olvidar, que sigue siendo el software específico de las asignaturas el encargado de crear y proporcionar gran parte de ese conocimiento reflexivo y profundo en los estudiantes. El objetivo del presente trabajo académico es mostrar una experiencia interdisciplinar de aplicación de las tecnologías TIC y recursos multimedia como complemento a programas de software específico dentro de un enfoque pedagógico Flipped Classroom.

\section{Objetivos}

En el presente documento se presenta una experiencia de innovación docente con carácter interdisciplinar. La experiencia está basada en la aplicación del enfoque pedagógico

(c)) BY-NC-ND 2018, Universitat Politècnica de València

Congreso IN-RED (2018) 
Flipped Classroom junto con la incorporación de diversas actividades activas-colaborativas desarrolladas dentro y fuera del aula durante el curso académico 2016/17. El ensayo educativo fue afrontado desde una perspectiva de investigación-acción en equipo docente. Las actividades pedagógicas del equipo docente universitario estuvieron orientadas a:

- Uso de nuevas aplicaciones, herramientas activas y mecanismos para la supervisión continua del estudiante. Facilitar la comprensión de conceptos y la adquisición de habilidades, destrezas y competencias asociadas a cada disciplina de conocimiento.

- Estructurar diferentes actividades académicas para desarrollarse dentro y fuera del aula (seminar y webinar). Motivar a los estudiantes durante el desarrollo de sus diferentes tareas académicas.

- Mejorar el acceso a recursos multimedia, archivos, materiales y documentación a lo largo del curso. Complementar el proceso de enseñanza-aprendizaje mediante el uso de sistemas online para promover un aprendizaje más cooperativo, reflexivo y significativo.

\section{Desarrollo de la innovación}

Se presenta una experiencia interdisciplinar de innovación educativa y renovación metodológica cuyo objetivo ha sido la incorporación del enfoque pedagógico Flipped Classroom en el aula. La implementación de nuevas herramientas TIC durante el proceso educativo ha permitido garantizar el éxito de la experiencia. En las asignaturas se maneja diferente software específico complementado con herramientas TIC gratuitas mediante el uso de smartphones, tablets y ordenadores portátiles, analizando su repercusión dentro y fuera del aula. Como conclusión del uso de los distintos recursos se puede afirmar que la actuación llevada a cabo ha resultado muy positiva para el alumnado, el profesorado y las asignaturas implicadas.

Los profesores implicados en la experiencia pertenecen a diferentes centros y áreas de conocimiento de la Universidad de Zaragoza: Área de Máquinas y Motores Térmicos, Área de Organización de Empresas, Área de Ingeniería Eléctrica o el Área de Anatomía y Embriología Humana lo que ha garantizado ese carácter interdisciplinar tan necesario en la implementación de experiencias de innovación educativa. A continuación se presentan las diferentes experiencias llevadas a cabo con software específico, complementado a su vez con múltiples recursos multimedia y herramientas TIC. En todos los supuestos el propósito ha sido alcanzar los objetivos iniciales en las asignaturas implicadas consiguiendo a su vez un aprendizaje más reflexivo, dinámico y significativo en el estudiante.

\subsection{Experiencia dentro del Área de Máquinas y Motores Térmicos.}

Los profesores del área de Máquinas y Motores Térmicos imparten asignaturas propias de la Ingeniería Térmica. En ellas se utilizan diversos programas específicos, además de las típicas hojas de cálculo, para el análisis, el dimensionamiento y la optimización de instalaciones energéticas. Por ser los más utilizados, aquí se presentan y comparan dos de ellos: Engineering Equation Solver (EES) y TermoGraf (Peña et al., 2017) 
El primero, EES (Fchart, 2018), se trata de un programa comercial para resolver sistemas de ecuaciones, con la ventaja de que incluye las funciones necesarias para calcular propiedades termodinámicas y para obtener otros parámetros importantes en el diseño de intercambiadores de calor, que de otra manera habría que obtener con tablas o modelos. En la figura 1 se muestra un ejemplo de programa junto con la solución obtenida. Este programa, de fácil manejo pero potente, resulta útil tanto a nivel educativo como a nivel profesional, aunque no es software libre y se debe pagar una licencia en función del uso.

Este programa se utiliza prácticamente en todas las asignaturas impartidas por el área tanto para las prácticas, como para trabajos tutorizados y actividades de evaluación. También es un programa muy utilizado en trabajos fin de estudios (TFG o TFM), ya que además de ser un software sencillo que resuelve ecuaciones, pueden hacerse simulaciones completas de instalaciones energéticas, a través de ventanas interactivas y diagramas de ciclos, figura 2.

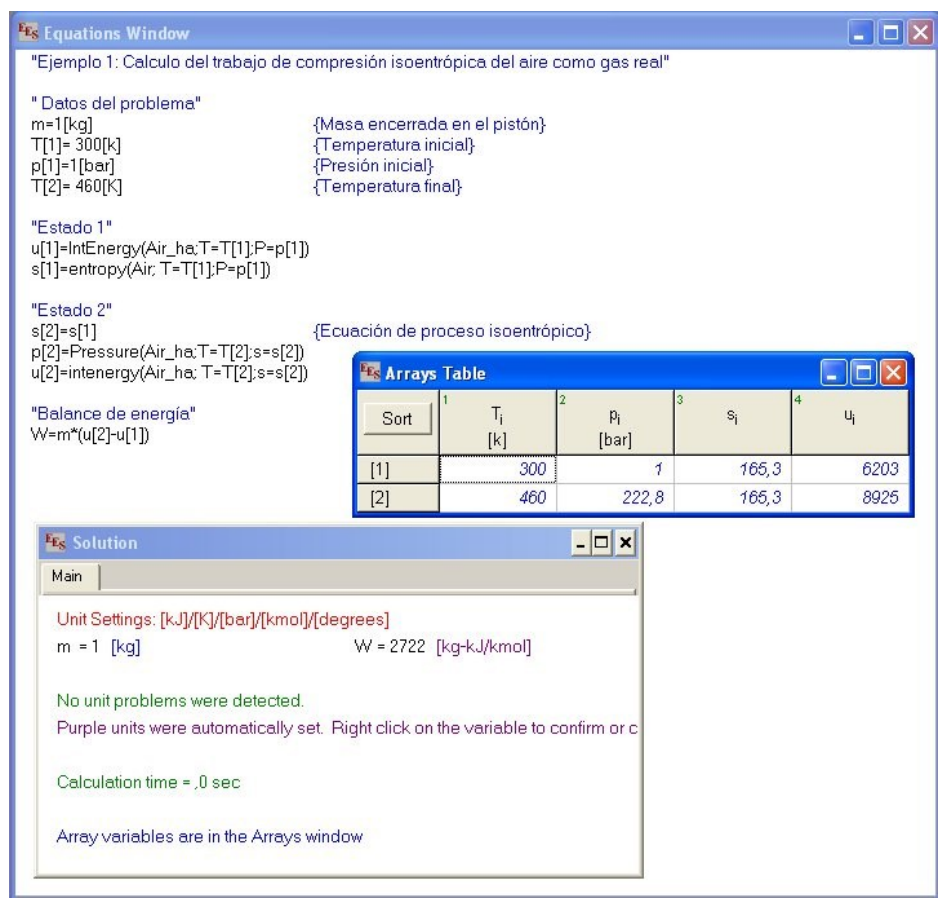

Fig. 1. Ejemplo de programa desarrollado en EES y la solución calculada.

El segundo programa, TermoGraf (TermoGraf, 2018), es un programa de software libre desarrollado en el área de Máquinas y Motores Térmicos de la Universidad de Zaragoza. Además, recientemente se ha desarrollado una aplicación para el móvil, Thermonator, que puede utilizarse como una calculadora para problemas de termodinámica. Su principal ventaja, además de que es completamente gratuito, es su enfoque eminentemente educativo: su uso es muy intuitivo y puede utilizarse en clase para generar fácilmente diagramas de propiedades en los que representar procesos y ciclos.

Este software tiene la ventaja de poder preparar lo que se denomina multiejercicios, que consisten en ejercicios con casos múltiples, es decir, con datos diferentes para cada estudiante. Cuando el estudiante accede con su número personal se le asigna un caso

(c)) EY-NC-ND 2018, Universitat Politècnica de València 
específico, cuya corrección es automática si se dispone de la licencia de profesor. Esto reduce la copia y el tiempo invertido en la corrección, aunque al no entregar la resolución completa no se puede identificar cuál ha sido el error cometido por el estudiante. Debido a los errores propagados no se puede evaluar si los cálculos posteriores son correctos o no; de esta forma el trabajo del estudiante puede estar minusvalorado. La desventaja principal es que su alcance está muy focalizado en termodinámica y no resulta tan útil en otras asignaturas en las que se involucra a otras disciplinas como transferencia de calor o termotecnia. Por esta razón, este software se utiliza esencialmente en la asignatura de Termodinámica Técnica, que es común a varios grados.

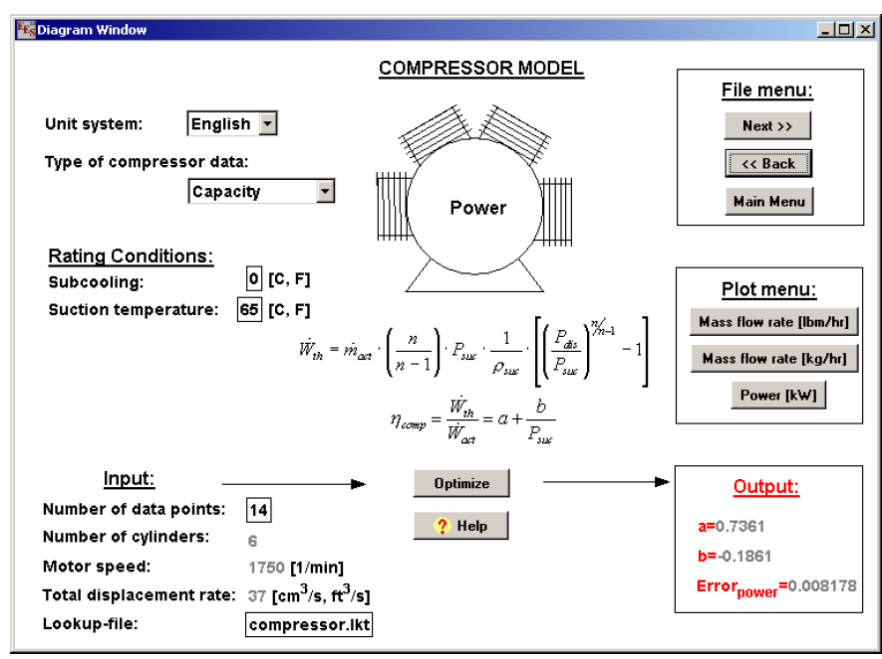

Fig. 2. Diagramas de ciclos y ventanas interactivas en EES (Manual de EES en (Fchart, 2018)).

En este trabajo se presentan las experiencias principalmente desarrolladas con el programa EES, por ser aplicable a problemas más generales. Concretamente, se muestra cómo ciertas herramientas TIC se combinan con el uso del programa para preparar las sesiones de prácticas y laboratorio. De esta manera se fomenta que el estudiante lleve la asignatura al día. En las sesiones de prácticas se resuelven con EES problemas de cierta complejidad, como calcular las prestaciones de un ciclo termodinámico completo y analizar el efecto de variar ciertos parámetros relevantes. Para la primera sesión, los estudiantes no conocen de antemano el manejo del programa, lo que ralentiza el desarrollo de la práctica y hace que, si no se toman medidas adicionales, los alumnos deban terminar los cálculos en casa, con el consiguiente riesgo de copia y de que queden dudas sin resolver. Por esta razón, se han utilizado algunas TIC que permitan al estudiante preparar la práctica, aprovechar mejor el tiempo en el aula y terminar el trabajo.

Por un lado, para la preparación de cada práctica y para aprender el manejo del programa EES, se han puesto a disposición de los estudiantes videos desarrollados por profesores del Área de Máquinas y Motores Térmicos dentro del proyecto PIIDUZ_15_079 (Zabalza, 2016-2017). De esta forma, el alumno puede visualizarlos tantas veces como necesite, puede practicar con el programa antes de la primera práctica y recopilar dudas concretas que puede resolver con el profesor al comienzo de la sesión. Para el presente curso además, 
se han implementado cuestionarios de autoevaluación para que el alumno pueda autoevaluarse de los contenidos de cada video.

Con respecto a las actividades de aprendizaje y evaluación, se han utilizado diversas metodologías y recursos. Tras finalizar cada tema, el alumno dispone de alrededor de una semana para resolver un cuestionario en Moodle sobre la parte teórica y un trabajo consistente en problemas de tipo examen que puede resolver con el programa EES. Durante el presente curso además, las soluciones de estos trabajos deben introducirse en Moodle para una corrección automática.

\subsection{Experiencia en el Área de Organización de Empresas.}

Dentro del ámbito de la docencia en administración y dirección de empresas son diversos los softwares utilizados. Por una parte, son destacables los simuladores de gestión empresarial integral, a modo de "juego de empresas", donde los alumnos interactúan en un contexto de competencia con otros participantes y toman decisiones encaminadas a aplicar los conocimientos adquiridos a lo largo de la titulación, para mejorar los resultados económicos de la empresa y en última instancia su supervivencia (Delgado et al., 2007). Por otra parte, existen softwares específicos útiles para facilitar la adquisición de diversas competencias en las distintas materias de los diferentes planes de estudios, ya se trate de programas para la gestión de inventarios, para el análisis estadístico, para la gestión contable o para la elaboración de modelos de negocio, entre otros. En este documento se presenta la experiencia correspondiente al uso de dos softwares específicos en "Dirección de Recursos Humanos", asignatura perteneciente al Grado en Administración y Dirección de Empresas, en la Facultad de Ciencias Sociales y Humanas.

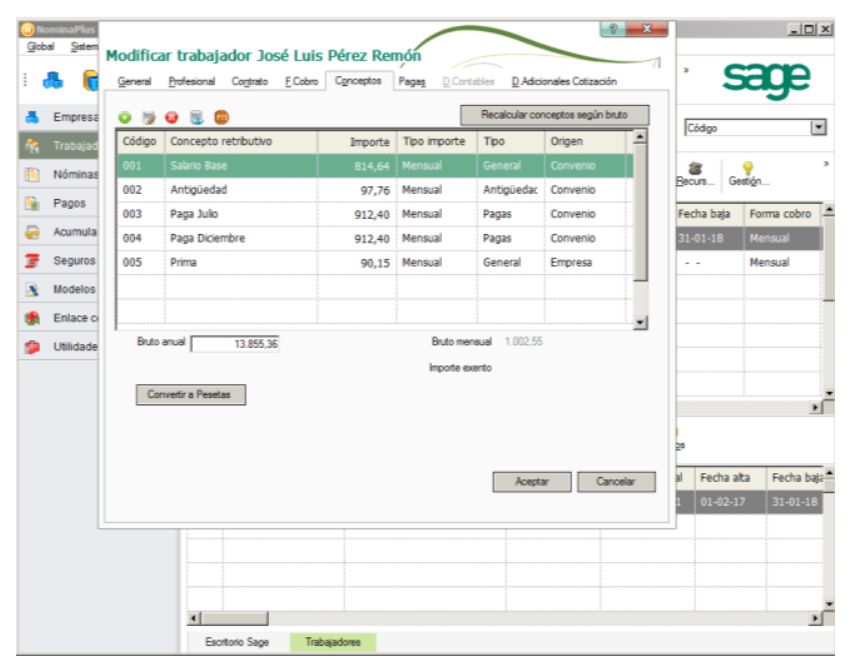

Fig. 3. Ejemplo del interface de usuario del software NominaPlus.

Dentro del ámbito de los recursos humanos existen gran variedad de programas dirigidos a mejorar la eficiencia empresarial. Estos programas suelen cubrir un amplio abanico de funciones como la gestión del personal, de los programas de formación, de los salarios, de

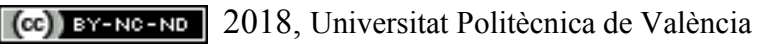

Congreso IN-RED (2018) 
los procesos de selección, etc. Existen tanto programas que integran las funcionalidades de recursos humanos a través de extensiones dentro de un software principal, incluido normalmente un sistema de planificación de recursos empresariales o ERP (Enterprise Resource Planning), como programas específicos de recursos humanos diseñados como soluciones independientes sin necesidad de integrarse en un programa mayor.

Por otra parte, puede distinguirse entre aquellos programas que requieren una licencia para su uso y aquellos programas gratuitos que permiten a las empresas de reducida dimensión acceder a utilidades diseñadas para la gestión de recursos humanos a un coste reducido o incluso nulo para un uso básico, pudiéndose completar con las versiones premium. De este modo también es relevante y destacable la aplicación de programas específicos de recursos humanos. Así pues se dan a conocer a los alumnos tanto programas gratuitos para la gestión de recursos humanos, como un programa con licencia para el cual la universidad dispone de permiso de uso. De esta manera el alumno conoce dos opciones diferentes y puede comprobar las utilidades ofrecidas por cada una de ellas. A continuación se pasa a describir brevemente cada uno de ellos:

Nominaplus. Se trata de un programa ampliamente utilizado por las empresas, ofrecido bajo licencia por el Grupo SAGE y para el cual la Facultad de Ciencias Sociales y Humanas de la Universidad de Zaragoza dispone de una licencia especial de uso educativo, figura 3. Su funcionalidad principal es la de un programa de nóminas que permite gestionar la relación con los empleados, las incidencias, el cálculo de los recibos de salarios, la liquidación de impuestos y seguros sociales, etc. Este programa presenta grandes similitudes con otros productos de la compañía como Contaplus o Facturaplus, con los que los alumnos pueden tener contacto en otras asignaturas o en posteriores cursos de especialización.

Factorial. Es un programa de creación reciente, que está destinado a automatizar las tareas del departamento de RRHH de las pequeñas y medianas empresas. En su versión gratuita, que es con la que se trabaja en clase, permite realizar distintas actividades relacionadas con la gestión documental, la obtención de informes, etc. y permite disponer de toda la información en la nube. Las funciones básicas son ampliadas en su versión premium.

Tal y como se recoge en la guía de la asignatura, la utilización del laboratorio y de herramientas TIC constituye una de las metodologías de enseñanza-aprendizaje aplicables a la asignatura Dirección de Recursos Humanos. Los objetivos que se persiguen con la utilización de los software anteriormente indicados son varios. Por una parte, se desea que el alumno sea conocedor de la existencia de estas herramientas, de las ventajas e inconvenientes que presenta cada una de ellas para la gestión de recursos humanos en las empresas y que se familiarice con su funcionamiento e interface. Por otra parte, estas actividades permiten consolidar los contenidos y competencias recogidos en la guía de la asignatura, complementando las actividades llevadas a cabo durante las clases de teoría y el resto de sesiones prácticas. Además, mediante el uso del software específico se fomentan las competencias relacionadas con la capacidad de análisis y el manejo de herramientas informáticas. 
El modo y momento de utilización varía dependiendo del software. En el caso del software Nominaplus, se realizan prácticas adicionales para completar un bloque concreto del temario, correspondiente a las retribuciones. En este caso únicamente se utiliza una parte de las utilidades que ofrece el programa, pues gran parte de ellas quedan fuera del alcance de la asignatura. En el caso del software Factorial, como se ha indicado, engloba un conjunto de funciones generales del departamento de RRHH, por lo que se utiliza en la gestión de los recursos humanos. En este caso las sesiones permiten al alumno iniciarse en el manejo de un programa generalista. Como se trata de un software gratuito, el alumno puede trabajar fuera del aula, de manera que las sesiones presenciales se complementan con el trabajo autónomo del alumno.

Ahora bien, ¿qué actividades desarrollan los estudiantes? La utilización de los software indicados se realiza fundamentalmente en las aulas de informática del centro. En el caso del software con licencia esta es la única opción posible. Sin embargo, en el caso del software libre, los alumnos pueden trabajar también fuera del aula, por lo que algunas de las actividades se planifican teniendo en cuenta estas circunstancias. En ambos casos los alumnos deben comenzar el proceso con la creación de una empresa en la aplicación, y la incorporación o alta de los distintos trabajadores y condiciones a aplicar, calendario laboral, etc. Posteriormente, los alumnos deben resolver los casos prácticos facilitados por el profesor. La corrección se lleva a cabo en el aula, de manera que el profesor puede indicar al alumno donde se han producido los fallos. Las prácticas se diseñan para favorecer el logro de los objetivos de aprendizaje y la adquisición de competencias.

Como ventaja principal destaca la oportunidad de vincular los conocimientos teóricos vistos en la asignatura y los ejercicios prácticos tradicionales o "en papel", especialmente los relativos al módulo correspondiente a las retribuciones, con la utilización de herramientas informáticas propias de los departamentos de recursos humanos. Esto influye positivamente en la motivación del alumnado, que percibe una mayor relación entre los conceptos vistos en la asignatura y la práctica empresarial real, lo que favorece la implicación del alumno. Sin embargo, la dificultad principal reside en la necesidad de encajar la utilización de dicho software con las disponibilidades de tiempo para el desarrollo de la asignatura.

\subsection{Experiencia en el Área de Ingeniería Eléctrica.}

En la última década, ha surgido un nuevo concepto dentro del escenario de la tecnología educativa: Simulation-based Learning (SbL). La mayoría de los educadores en ciencias e ingeniería creen que la experiencia en el laboratorio es un complemento necesario frente a otro tipo de actividades pasivas, como leer libros de texto y escuchar conferencias; esta serie de actividades constituye una gran parte del tiempo disponible (Rutten et al., 2012). Hoy en día, los métodos de aprendizaje en las universidades se encuentran en constante proceso de revisión. Aplicaciones enfocadas al SbL han permitido acelerar el proceso de aprendizaje (Artal-Sevil et al., 2015), facilitando el diseño e implementación de los recursos interactivos en el aula. Simulation-based Learning o Learning by-doing son técnicas muy efectivas para que los estudiantes adquieran conocimientos, si están bien motivados. Estos

(c)) BY-NC-ND 2018, Universitat Politècnica de València

Congreso IN-RED (2018) 
modelos de aprendizaje no son nuevos, y permiten integrar simultáneamente las ventajas del método e-learning (recursos multimedia, simulaciones interactivas, webinar, etc.) con aspectos relacionados con el método tradicional (interacción face-to-face).
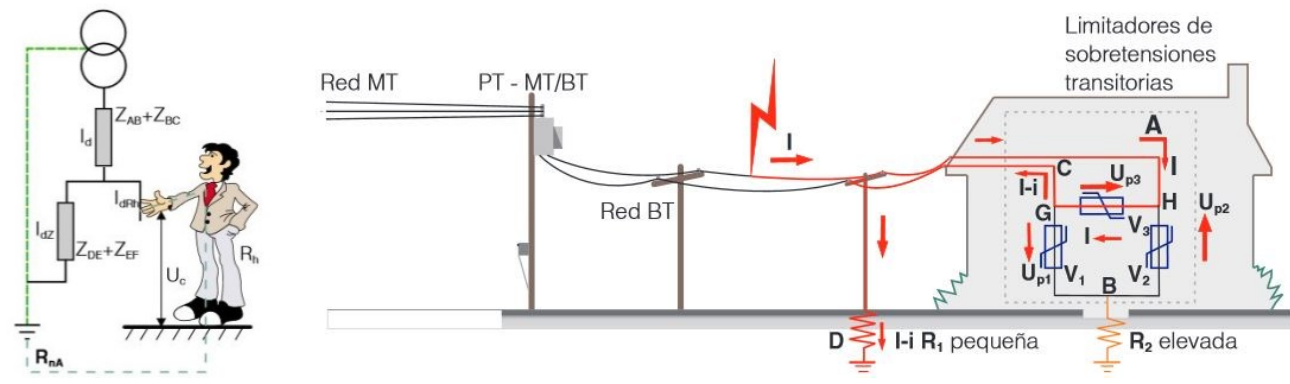

Fig. 4. Ejemplo de software de simulación e interface gráfico. Comprensión de los conceptos de corrientes de fuga en distribución y sistemas de protección diferencial.

La implementación de esta serie de técnicas educativas permite al instructor tener una visión más amplia de los conocimientos y destrezas adquiridas por los estudiantes durante el cuatrimestre académico. Debe notarse que el profesor tiene un feedback importante sobre la adquisición de conocimiento de los estudiantes. Por otra parte, este tipo de metodología docente permite desarrollar un método de evaluación más amplio, en comparación con otros métodos más tradicionales. Así pues es posible identificar no sólo el conocimiento del estudiante, ya que se cuestiona, analiza, sintetiza,... sino verificar también el proceso de asimilación de contenidos. Desde el punto de vista del profesor la técnica SbL es valorada positivamente, ya que ofrece una visión reflexiva e interactiva de la educación.

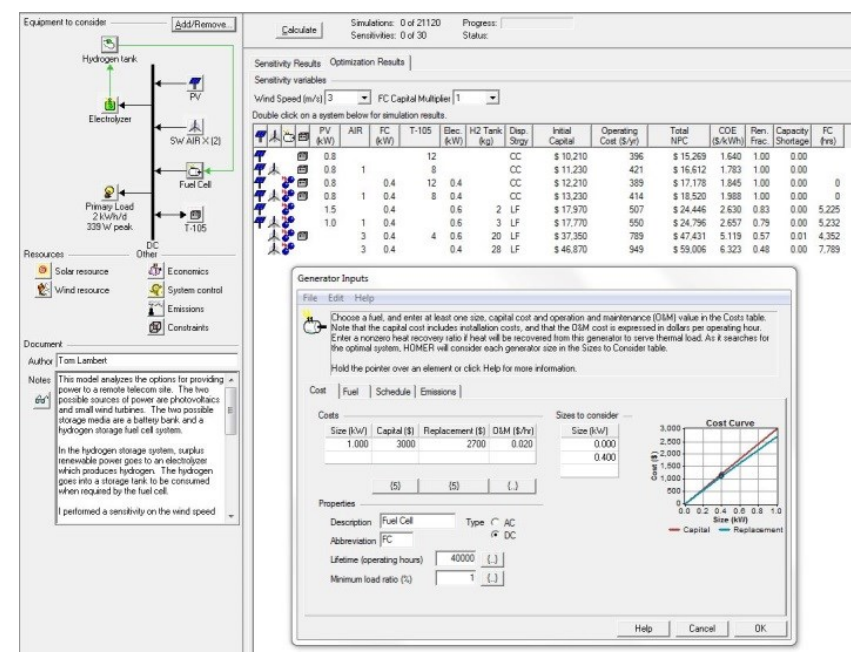

Fig. 5. Ejemplo del software de simulación utilizado en Ingeniería y su interface gráfico. Software educacional versión-demo del programa HOMER Renewable Energy.

La figura 5 muestra un ejemplo de software de simulación destinado al análisis y cálculo de sistemas híbridos de energía renovable. HOMER es un software para microrredes y diseño y optimización de sistemas de generación distribuida. La aplicación también puede 
incorporar las curvas de recursos renovables para realizar las estimaciones necesarias. Se puede descargar en la dirección web http://www.homerenergy.com/.

Este tipo de herramientas se han convertido en importantes recursos educativos. El cálculo, la estimación y el desarrollo de los diferentes modelos tecnológicos son actividades que los estudiantes pueden realizar fuera del aula, de modo que se optimiza el tiempo de clase (enfoque pedagógico Flipped Classroom). Así pues, el aula se transforma en un seminario para responder a diversas preguntas asociadas con el análisis del modelo (parámetros o coeficientes de simulación, configuraciones, tiempo de respuesta, etc.) y no asociadas al entorno del software de simulación usado.

Por lo tanto, si el objetivo es ayudar a los estudiantes a familiarizarse con el diseño y dimensionamiento de instalaciones e implementación de sistemas en el mundo real, el uso de estas tecnologías se hace inevitable. Asimismo ofrecen un terreno fértil para desarrollar nuevas técnicas innovadoras con objeto de involucrar e inspirar al estudiante. La satisfacción general de los estudiantes con respecto a los métodos de enseñanza adoptados y las técnicas aplicadas es muy buena. También debe tenerse en consideración que este procedimiento requiere mayor atención por parte del docente debido a la necesidad de verificar y revisar todos los modelos y simulaciones efectuados por cada estudiante.

Las fases iniciales han permitido que los alumnos puedan aplicar los conocimientos y contenidos adquiridos. Al mismo tiempo, han aprendido a superar las dificultades inherentes al proceso de creación, cálculo y diseño de los modelos planteados. En general, los modelos de simulación desarrollados por los estudiantes, que combinan aspectos técnicos y teóricos, tienen una calidad aceptable. Además, se ha demostrado que el uso de estas herramientas ha introducido un aprendizaje más significativo. El factor de curiosidad que representa el uso del software de simulación (no hay que olvidar que está vinculado con problemas reales de ingeniería) facilita una buena recepción por parte de los estudiantes.

En el caso analizado en este epígrafe, el curso se encuentra soportado por abundantes recursos multimedia junto con otras demostraciones y tutoriales en video. Las diferentes actividades académicas desarrolladas por los estudiantes son debidamente documentadas (aplicación de la técnica e-portfolio). Se han utilizado varias herramientas TIC como complemento al software específico, con el propósito de obtener un feedback inmediato. Por otra parte la plataforma educativa Moodle ha sido usada como repositorio de información y contenidos para los estudiantes.

\subsection{Experiencia dentro del Área de Anatomía y Embriología Humana.}

La enseñanza práctica de la asignatura "Anatomía e Histología ocular”, cursada en el primer año académico en el Grado de Óptica-Optometría, se basa en el aprendizaje cooperativo, para ello los alumnos se dividen en grupos pequeños liderados por uno de ellos al que se le denomina jefe de mesa o alumno monitor, sobre el que recae la responsabilidad de la práctica. Este rol va rotando en las diferentes prácticas realizadas durante el año académico. Para acompañar a los alumnos tanto en su aprendizaje autónomo como en la

(c)) BY-NC-ND 2018, Universitat Politècnica de València

Congreso IN-RED (2018) 
responsabilidad de dirigir a sus compañeros en la labor que le ha sido asignada, se ha elaborado un material docente-guía para las sesiones prácticas (Luesma et al., 2013).

El aprendizaje electrónico (u-learning), especialmente en el ámbito de la educación biomédica, permite el acceso a la información, al entreno con gran inmediatez y una actualización continuada (Grau-Perejoan, 2008), siendo un medio tan efectivo para la educación sanitaria como los sistemas tradicionales (Wutho et al., 2004). Si además, la enseñanza se realiza de manera compatible con el estilo de aprendizaje de los estudiantes, éstos aprenderán mejor, retendrán más ese conocimiento y tendrán una actitud más positiva frente al objeto de estudio.

Según el modelo de la Programación Neurolingüística de Bandler y Grinder (1988), también llamado visual-auditivo-kinestésico (VAK), tenemos tres grandes sistemas para representar mentalmente la información, relacionados directamente con los estilos de aprendizaje: el visual, el auditivo y el kinestésico. El docente debe de conocer el perfil de sus estudiantes y las singularidades de sus disciplinas, seleccionando la modalidad sensorial que mejor se adapte para el proceso enseñanza-aprendizaje y diseñando actividades adaptadas a cada estilo. Se cree que el $40 \%$ de los estudiantes presentan un aprendizaje fundamentalmente visual (Clark et al., 2006), aspecto que beneficia enormemente a la docencia de nuestra asignatura, altamente visual, al ser muy descriptiva y basada en la observación directa de la naturaleza de nuestro organismo.
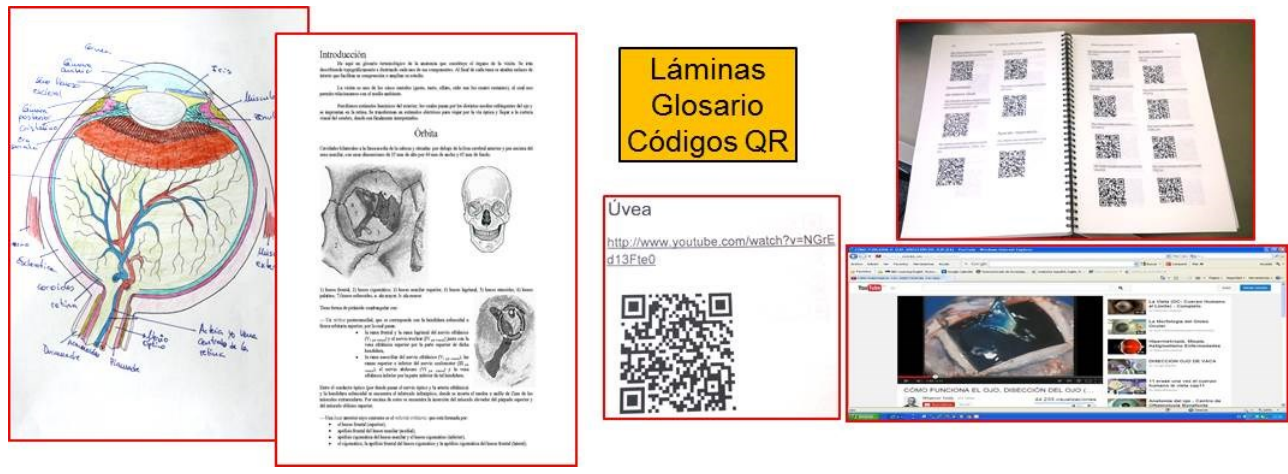

Fig. 6. Ejemplo de diferentes materiales utilizados por los estudiantes en el contexto señalado.

Por otra parte, uno de los problemas al que se suele enfrentar el alumno de nuevo ingreso en una Facultad en el ámbito de la Profesión Sanitaria, es abordar un vocabulario complejo, muy técnico y nuevo para él, de hecho, suele ser una de las mayores barreras para el aprendizaje de las ciencias, incluidas las biomédicas (Wellington y Osbourne, 2001). Tal es el caso de la Anatomía e Histología. El profesor dentro de su rol de mediador entre la disciplina y los estudiantes debe de facilitar el canal adecuado con el fin de incrementar el rendimiento de los estudiantes; desarrollar herramientas visuales, interactivas y rápidas le va a ayudar a conseguir ese objetivo.

Valorando todos estos aspectos, fue diseñada una guía-manual, reinventando metodologías clásicas combinadas con tecnologías más modernas. Esta guía es un material interactivo que sirve de hoja de ruta tanto al instructor como a los otros miembros del grupo en cada sesión práctica. Recoge una colección de láminas que el alumno debe ilustrar y completar, 
y un apéndice-glosario terminológico que facilitará la resolución de dudas que puedan ir surgiendo en el transcurso de la unidad práctica de manera autónoma y sólida. Igualmente se proponen enlaces con páginas web y una colección de códigos QR (quick response code) de interés para cada una de las materias abordadas. Toda esta información puede ser consultada por los alumnos durante la realización de la práctica a través de sus dispositivos móviles (smartphone, tablets, etc.) gracias a herramientas digitales gratuitas.

Los códigos de respuesta rápida o código $\mathrm{QR}$, se caracterizan por almacenar gran cantidad de información gracias a una matriz de puntos. Para poder descifrar la información es necesario disponer de un lector de códigos QR, cuya aplicación se encuentra disponible para teléfonos móviles y tablets mediante dispositivos de captura de imagen, y una conexión a Internet para las direcciones web (http://www.qrcode.com/en/history/). Este material permite que los alumnos en la propia sala de disección identifiquen estructuras, consulten un glosario terminológico especializado o enlacen con web de interés en tiempo real. Es evidente que múltiples de sus sentidos se encuentran trabajando simultáneamente de manera individual y grupal, lo que además confiere un carácter lúdico que también es clave en el aprendizaje, ya que sirve de estrategia afectiva puesto que desinhibe, relaja y motiva. La gamificación favorece la memorización cuando el juego consiste en repetir una estructura o sistemas mnemotécnicos.

Según nuestra experiencia y a la luz de los resultados, el aprendizaje electrónico ha revelado ser un medio muy efectivo para la educación biosanitaria. Hay que destacar su gran utilidad para adquirir habilidades en las que el componente visual es predominante, tal es el caso de imágenes histológicas de tejidos orgánicos, disecciones cadavéricas, etc. tan necesarios en esta disciplina y que el alumno puede consultar on-line a través de la Bitácora a tiempo real mientras se está desarrollando la sesión práctica.

\section{Diferentes Herramientas TIC utilizadas}

En la actualidad la introducción de las herramientas TIC en la enseñanza ha proporcionado muchos beneficios sobre la educación universitaria. De esta forma ha sido posible facilitar el aprendizaje de los estudiantes, incrementar su interés y mejorar su rendimiento. Estos recursos tecnológicos se han convertido en un aliado poderoso para el profesor, pues introducen mejoras en el proceso enseñanza-aprendizaje. En la experiencia desarrollada aquí, se han utilizado diferentes herramientas TIC interactivas y recursos multimedia que han mejorado las sesiones de enseñanza; destaca el uso del software gratuito y libre. Como ejemplo, el instructor prepara una sesión con preguntas (Q\&A) relacionadas con los conocimientos básicos impartidos en el aula y cada estudiante utiliza su propio smartphone para responder a cada pregunta; los resultados son inmediatos. Profesor y estudiante obtienen un feedback inmediato. Además esta estrategia permite mejorar las actividades de aprendizaje ayudando al estudiante a aprender de una manera más divertida y entretenida.

Socrative, Quizizz o Kahoot son aplicaciones de software gratuito que permiten desarrollar cuestionarios en línea con el fin de que los estudiantes respondan en tiempo real a través de cualquier dispositivo móvil, convertido en un mando (iClickers). Los resultados se obtienen

(cc) EY-NC-ND 2018, Universitat Politècnica de València

Congreso IN-RED (2018) 
de inmediato y se pueden mostrar a los alumnos en tiempo real; de este modo constituyen buenos materiales interactivos. Estas herramientas son muy sencillas e intuitivas y sólo requieren de unos pocos segundos para iniciar la sesión. Las respuestas del alumnado y su puntuación se presentan visualmente a través de un gráfico o diagrama. Los estudiantes reciben feedback personal en su dispositivo.

Google-Apps (G-Suite) ofrece un conjunto de herramientas de gran alcance que se pueden utilizar para desarrollar entornos de colaboración entre los estudiantes. La plataforma cuenta con un grupo diverso de aplicaciones que pueden ser destinadas a la educación, tales como: Google-Drive, Google-Classroom, Gmail, Calendar, Docs, Google-Search, Forms, Hangouts, Slides, etc. Estas aplicaciones permiten la comunicación, almacenar información en la nube, desarrollar actividades de planificación, búsqueda de información, etc.

Evernote y OneNote son aplicaciones gratuitas que permiten la creación del e-portafolio, se utilizan principalmente para organizar la información a través de una libreta virtual, notebook. Incorporan una amplia gama de funciones y herramientas diversas. Su uso es relativamente simple e intuitivo. Permite mantener actualizadas y organizadas las tareas y notas relativas a la asignatura. También es posible desarrollar una evaluación formativa. El interface es simple y agradable, ya que está basado en un editor de texto básico. Los cuadernos personales son siempre privados y totalmente controlados por el usuario. En cada nota es posible incluir nuestras ideas, listas de tareas y recordatorios incorporando texto, imágenes, audio, dibujos, notas manuscritas, ecuaciones matemáticas, etc.

Screencast-O-Matic, Windows Movie Maker, EDpuzzle o Movenote son una serie de aplicaciones destinadas a la elaboración de materiales audiovisuales. Estos programas permiten la grabación de video-lecciones, fraccionar un vídeo e introducir un conjunto de preguntas sobre los diferentes fragmentos. Los estudiantes visualizan el vídeo con mayor atención, ya que tienen que responder a una serie de preguntas más adelante. Estas herramientas son muy versátiles, su uso es relativamente sencillo y no requiere un nivel de conocimientos profundo.

\section{Resultados y Conclusiones}

El desarrollo de esta experiencia ha permitido analizar la utilidad de la estrategia Flipped Classroom complementada por otra serie de técnicas y recursos en el contexto universitario. La tecnología y las actividades de aprendizaje se convierten en fundamentales en este nuevo modelo al que podríamos englobar en un tipo de Blended Learning, facilitado a través de la combinación eficiente de recursos virtuales y físicos. Este modelo es un enfoque integral que combina la instrucción directa del alumnado con métodos constructivistas. De este modo se han podido analizar las bondades y beneficios que ofrece esta nueva estrategia en los diversos procesos de enseñanza-aprendizaje.

Las diferentes acciones llevadas a cabo han mejorado el paradigma enseñanza-aprendizaje, fomentando la utilización de recursos educativos destinados a la innovación dentro y fuera del aula. El uso del software específico complementado con diferentes herramientas TIC gratuitas (Kahoot, Socrative, Quizizz, EverNote, EDPuzzle, QR-Codes,...) ha permitido 
crear cuestionarios interactivos (Q\&A), e-portfolio, material audiovisual, etc. fomentando los procedimientos de autoevaluación y coevaluación de los estudiantes. La plataforma Moodle2.0 ha sido utilizada en la mayoría de los casos como repositorio de información, archivos, videos, contenidos multimedia, etc., también ha permitido la entrega de diferentes actividades académicas así como la evaluación de los estudiantes a través de cuestionarios. Se ha podido comprobar que los alumnos valoran muy positivamente el uso del software específico en las diferentes asignaturas.

Así pues, el uso adecuado de las TIC en las diferentes asignaturas ha producido un estímulo sobre el alumno, mejorando la percepción ante sus tareas académicas. Como punto esencial innovador, se puede destacar la introducción de estas herramientas tecnológicas tan actuales y habituales en nuestra sociedad en el proceso de enseñanza-aprendizaje con fines realmente educativos, y su repercusión en los distintos niveles de enseñanza. También se ha observado como la introducción de esta estrategia de aprendizaje ha incrementado el grado de motivación del estudiante. Al mismo tiempo los estudiantes prefieren procedimientos de enseñanza-aprendizaje más dinámicos, flexibles, participativos y con evaluación continua. Estas herramientas educativas interactivas han incrementado las destrezas, habilidades y competencias de los estudiantes. La experiencia llevada a cabo nos muestra resultados positivos desde una triple vertiente: tanto para los estudiantes, como para el profesorado y para las propias asignaturas implicadas.

La experiencia de innovación docente aquí presentada es económicamente sostenible, eficiente y transferible a otras disciplinas de conocimientos. Diferentes estudios indican que la integración del Flipped Classroom junto con técnicas de e-learning pueden mejorar los resultados de los estudiantes, al mismo tiempo que se obtiene un aprendizaje más profundo y significativo. Así la metodología implementada ayuda a mejorar el aprendizaje del alumno. Los estudiantes han conseguido el mismo nivel de conocimiento que en cursos académicos anteriores con metodologías de aprendizaje más tradicionales. El grado de satisfacción de los estudiantes durante la experiencia ha sido alto, tal y como han revelado las encuestas de evaluación de la actividad docente en las diferentes asignaturas.

\section{Agradecimientos}

Los autores agradecen el apoyo financiero de la Universidad de Zaragoza para el desarrollo del presente trabajo, a través del Programa de Innovación e Investigación Educativa para Grupos de Profesores del Vicerrectorado de Política Académica de la Universidad de Zaragoza (PIIDUZ 2017/18). El trabajo aquí mostrado se ha desarrollado dentro del marco de los proyectos con los siguientes identificadores: PIIDUZ_17_299 y PIIDUZ_17_059.

\section{Referencias}

ARTAL-SEVIL, J.S., ROMERO E., and ARTACHO J.M. (2017a). Quick surveys in classroom. Mobile phone, a Powerful Teaching tool. 11th International Technology, Education and Development Conference, INTED17. IATED Digital Library. Valencia, Spain. 06-08 March 2017; pp.: 9282-9291. DOI: $10.21125 /$ inted.2017.2194

(cc) EY-NC-ND 2018, Universitat Politècnica de València

Congreso IN-RED (2018) 
ARTAL-SEVIL, J.S., LUESMA, M.J., GUILLEN-MONZON, N., and ROMERO-PASCUAL, E. (2017b). Addressing flipped classroom from several teaching methodologies. An interdisciplinary experience. 9th International Conference on Education and New Learning Technologies, EDULEARN'17. IATED Digital Library. 3-5 July, 2017 Barcelona, Spain; pp: 5051-5061. DOI:10.21125/edulearn.2017.2132

ARTAL-SEVIL, J.S., BERNAL-AGUSTIN, J.L., and DOMINGUEZ J.A. (2015). m-Learning (mobile Learning) in Education. The impact of smartphone as interactive learning tool. 7th International Conference on Education and New Learning Technologies, EduLearn15. IATED Digital Library. Barcelona, Spain. 06-08 July 2015; pp.: 8212-8221.

BANDLER, R. y GRINDER, J. (1988). Use su cabeza para variar. Seminario sobre técnicas de Programación Neurolingüística (PNL). Cuatro vientos, Santiago de Chile.

CLARKE, I., FLATHERTY, T. and YANKEY, M. (2006). Teaching the visual learner: The use of Visual summaries in Marketing Education. Journal of Marketing Education 28(3), 218-226.

DELGADO, J. M., ESPITIA, M. A., \& ROSELL, J. (2007). Experiencias docentes a partir de la gestión virtual de empresas. En: Innovación docente, tecnologías de la información y la comunicación e investigación educativa en la Universidad de Zaragoza: caminando hacia Europa (p. 66). Universidad de Zaragoza.

FCHART (2018) Engineering Equation Solver. F-Chart Software, LLC. <http://www.fchart.com/ees/> [Consulta: 17 de marzo de 2018].

GÁMEZ-MONTALVO, M.J. y TORRES-MARTÍN, C. (2013). Las técnicas de grupo como estrategia metodológica en la adquisición de la competencia de trabajo en equipo de los alumnos universitarios. Journal for Educators, Teachers and Trainers 4, 14-25.

GRAU-PEREJOAN, Ov. (2008). Formación on-line. Educación Médica 11(3), 139-146.

JONSSON, H. (2015). Using flipped classroom, peer discussion, and just-in-time teaching to increase learning in a programming course. Frontiers in Education Conference (FIE 2015). IEEExplore Digital Library. Recuperado el 02/10/16 de http://ieeexplore.ieee.org/document/7344221/

LUESMA BARTOLOMÉ, M.J. y FRANCO BALLESTÍN, A. (2013). Bitácora Anatómica e Histológica Ocular. Prensas Universitarias de Zaragoza, España.

PEÑA, B. et al. (2017). Pilot experience for the application of the flipped classroom in subjects of the field of Thermal Engineering. En: INTED17 Proceedings of the 11th annual International Technology, Education and Development Conference, Valencia, IATED Academy, pp. 3601-3610. DOI: 10.21125/inted.2017.0887.

RUTTEN N., van JOOLINGEN W.R. and van der VEEN J.T. (2012). The learning effects of computer simulations in science education. Elsevier ScienceDirect. Computers \& Education. Volume 58, Issue 1; pp 136 to 153.

TERMOGRAF (2018) TermoGraf, simulador termodinámico. Universidad de Zaragoza. $<$ http://termograf.unizar.es/www/index.htm $>$ [Consulta: 17 de marzo de 2018].

TOPPO, G (2011). Flipped Classrooms take advantage of technology. USA Today, July 10. Recuperado el 04/10/16 de http://usatoday30.usatoday.com/news/education/story/2011-10-06/flipped-classroomsvirtual-teaching/50681482/1

TOURÓN, J., SANTIAGO, R., \& DÍEZ, A. (2014). The Flipped Classroom. Cómo convertir la escuela en un espacio de aprendizaje. Barcelona: Digital-text.

WELLINGTON, J. and OSBOURNE, J. (2001). Language and literacy in science education. Philadelphia, PA: Open University press.

WUTOH, R., BOREN, S.A. and BALAS, E.A. (2004). e-Learning: A Review of Internet-Based Continuing Medical Education. The Journal of Continung Education in the Health Professions 24(1), 20-30.

ZABALZA, I., PEÑA, B., LLERA, E.M. and USÓN, S. (2016) "Improving the teaching-learning process using educational videos as reusable learning objects in the field of thermal engineering", Proceedings of the 8th International Conference on Education and New Learning Technologies (EDULEARN 2016), Barcelona, IATED Academy, pp. 363-372. DOI: 10.21125/edulearn.2016.1068.

(c) ) EY-NC-ND 2018, Universitat Politècnica de València 